\section{Pertuzumab nun zugelassen - Meilenstein für die First-Line-Therapie des metastasierten HER2-positiven Mammakarzinoms}

Seit März 2013 ist Pertuzumab (Perjeta ${ }^{\circledR}$ ) in Kombination mit dem bisherigen Therapiestandard Trastuzumab $\left(\right.$ Herceptin $\left.^{\circledR}\right)$ plus Docetaxel für die First-Line-Therapie des metastasierten HER2-positiven Mammakarzinoms zugelassen. In der Behandlung dieser schweren Tumorerkrankung leitet dies eine neue Ära ein: Der humanisierte monoklonale Antikörper ermöglicht eine bislang einzigartige zielgerichtete Therapie, die den betroffenen Frauen die Aussicht auf ein signifikant verlängertes Gesamtüberleben (OS) eröffnet [1]. Anlässlich der Zulassung erläuterten Privatdozentin Dr. Brigitte Rack, München, und Professor Dr. Andreas Schneeweiss, Heidelberg, in einer Pressekonferenz der Roche Pharma AG den hohen Stellenwert der neuen Therapieoption und den einzigartigen Wirkmechanismus des HER2-Dimerisierungs-Inhibitors Pertuzumab.

Bei etwa jeder fünften Patientin mit Brustkrebs wird HER2 auf der Oberfläche der Tumorzellen überexprimiert [2]. Aufgrund eines besonders aggressiven Tumorverhaltens galt der positive HER2-Status lange als ein äußerst negativer prognostischer Faktor [3]. Die Zulassung des ersten gegen HER2-gerichteten Antikörpers Trastuzumab im Jahr 2000 hat die Prognose der betroffenen Frauen entscheidend verbessert: Heute ist der positive Rezeptorstatus mit einem signifikanten Überlebensvorteil assoziiert [4]. Dennoch schreitet die Erkrankung bei jeder zweiten Patientin innerhalb eines Jahres voran. «Gerade für Frauen mit einer metastasierten Brustkrebserkrankung haben wir einen dringenden Bedarf an neuen, HER2-spezifischen Therapieoptionen», betonte Rack. «Die Zulassung von Pertuzumab ist daher für die klinische Praxis von höchster Relevanz. Mit der zusätzlichen Gabe von Pertuzumab zum bisherigen Standard Trastuzumab plus Docetaxel können wir unsere Patientinnen erheblich effektiver behandeln - und dies nicht auf Kosten einer gesteigerten Toxizität.»

\section{CLEOPATRA-Studie:}

Signifikanter Überlebensvorteil mit Pertuzumab

Die Ergebnisse der randomisierten, Placebokontrollierten Phase-III-Zulassungsstudie CLEOPATRA belegen die Vorteile der neuen Behandlungsstrategie: Bei zuvor gegen die metastasierte Erkrankung nicht behandelten Patientinnen reduzierte Pertuzumab in Kombination mit Trastuzumab plus Docetaxel das Sterberisiko hochsignifikant um 34\% ( $\mathrm{HR}=0,66$; $\mathrm{p}=0,0008$ ). Während der Median im Kontrollarm bei 37,6 Monaten lag, war er zum Zeitpunkt dieser OS-Analyse im Pertuzumab-Arm noch nicht erreicht, da über die Hälfte der Patientinnen noch lebte [1]. Auch hinsichtlich der weiteren zentralen klinischen Endpunkte war das Pertuzumab-Regime dem bisherigen Standard überlegen: So verlängerte Pertuzumab das progressionsfreie Überleben (PFS) von median 12,4 auf 18,5 Monate ( $\mathrm{HR}=0,62 ; \mathrm{p}<0,0001)$; die objektive Ansprechrate (ORR) verbesserte sich von 69,3 auf $80,2 \%(p=0,0011)$ [5]. Gleichzeitig erwies sich die zusätzliche Behandlung mit Pertuzumab als sicher und gut verträglich: Die Abbruchraten im PertuzumabArm waren nahezu identisch mit denen im Kontrollarm (6,1 vs. 5,3\%). Nebenwirkungen $\geq$ Grad 3 traten fast ausschließlich während der initialen Kombination mit der TaxanChemotherapie auf [6]. «Die Ergebnisse der CLEOPATRA-Studie belegen eindeutig die hohe Wirksamkeit von Pertuzumab», so das Fazit von Schneeweiss. «Mit der Kombination Pertuzumab plus Trastuzumab plus Docetaxel haben wir einen neuen Standard für die FirstLine-Therapie des metastasierten HER2-positiven Mammakarzinoms.»

\section{Einzigartiger Wirkmechanismus:}

HER2-Dimerisierungs-Inhibition

Die hohe Wirksamkeit des Pertuzumab-basierten Therapieregimes beim HER2-positiven Mammakarzinom beruht auf dem einzigartigen Wirkmechanismus der Substanz: Mit Pertuzumab wurde erstmals ein Medikament entwickelt, das speziell die Dimerisierung des HER2-Rezeptors mit anderen Mitgliedern der HER-Familie - insbesondere HER3 - unterbindet. Dadurch blockiert Pertuzumab gezielt Wachstumsimpulse, die für die Tumorprogression von zentraler Bedeutung sind. «Dieser Wirkmechanismus der HER2-DimerisierungsInhibition ist komplementär zu dem von Trastuzumab», erklärte Schneeweiss. «Die Kombination Pertuzumab plus Trastuzumab ermöglicht daher, HER2-spezifische Tumorsignale noch effektiver zu blockieren».

\section{Referenzen \\ Swain S et al.: SABCS 2012; Poster \# P5-18-26. 2 Wolff AC et al.: Arch Pathol Lab Med 2007;131. 3 Slamon DJ et al.: Science 1989;244:707-712. \\ 4 Dawood S et al.: J Clin Oncol 2010;28:92-98. \\ Baselga J et al.: N Engl J Med 2012;366:109-119. \\ 6 Baselga J et al.: J Clin Oncol 2012;30(suppl), abstr \# 597}

Weitere Informationen bei

Roche Pharma AG

Ulla Satzger

Emil-Barell-Straße 1, 79639 Grenzach-Wyhlen ulla.satzger@roche.com

www.roche.com

\title{
PharmaTicker+++ PharmaTicker+++ PharmaTicker+++ PharmaTicker+++
}

Genomic Health. Das National Institute for Health and Clinical Excellence (NICE) hat eine überarbeitete Version des Dokuments zur diagnostischen Beratung bei genomischen Tests herausgegeben. In diesem Entwurf wird der Oncotype $\mathrm{DX}{ }^{\circledR}$ Test als einziger MultigenBrustkrebstest für die klinische Anwendung bei einigen Patientinnen mit Östrogen-positivem, Lymphknoten-negativem und HER2-negativem, frühem Brustkrebs empfohlen, um die Entscheidung für oder gegen eine Chemotherapie zu treffen.

Genomic Health Deutschland GmbH

Dr. Gerald Wiegand

gwiegand@genomichealth.com sphingotec GmbH. Für gesunde Frauen, bei denen eine erhöhte Proneurotensin (pro-NT)Plasmakonzentration gemessen wird, besteht ein signifikant erhöhtes Risiko, an Brustkrebs zu erkranken. Im zweiten Quartal 2013 bringt das Brandenburger Unternehmen in Deutschland den Labortest sphingotest pro-NT auf den Markt, mit dem Frauen feststellen lassen können, ob ihr individuelles Risiko erhöht ist. Der Nachweis über die direkte Korrelation zwischen erhöhten pro-NT-Werten und Brustkrebs wurde über die retrospektive Analyse einer Populationsstudie erbracht.

sphingotec $\mathrm{GmbH}$

Dr. Ute Kilger

kilger@sphingotec.de
Radiologie und Nuklearmedizin Ludwigshafen. Die bestmögliche Brustkrebstherapie basiert u.a. auf der Genauigkeit des vorangehenden Diagnoseverfahrens. Bestehende Verfahren stoßen gerade bei der Erkennung und Diagnose kleinster Bereiche abnormalen Gewebes im Frühstadium an ihre Grenzen. US-Studien und die wissenschaftlichen Auswertungen von Dr. Müller, Ludwigshafen, weisen nach, dass die methodische Zuverlässigkeit der Positronen-Emissions-Mammografie (PEM)Methode zur Feststellung eines Brustkrebstumors (Spezifität und Sensivität) bei über $90 \%$ liegt. Dr. Frank Müller

Radiologie und Nuklearmedizin Ludwigshafen www. radiologie-ludwigshafen.de 